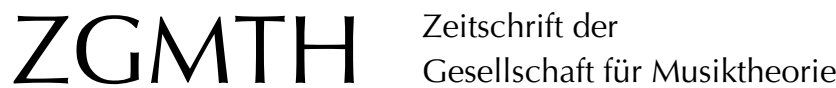

Ott, Immanuel (2012): Das kompositorische Verfahren in Jean Moutons Quadrupelkanon Nesciens mater virgo virum. ZGMTH 9/1, 13-23. https://doi.org/10.31751/671

(C) 2012 Immanuel Ott

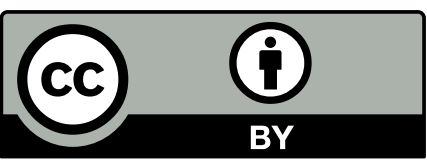

Dieser Text erscheint im Open Access und ist lizenziert unter einer Creative Commons Namensnennung 4.0 International Lizenz.

This is an open access article licensed under a

Creative Commons Attribution 4.0 International License.

veröffentlicht / first published: 26/11/2012

zuletzt geändert / last updated: 25/09/2012 


\title{
Das kompositorische Verfahren in Jean Moutons Quadrupelkanon Nesciens mater virgo virum
}

\author{
Immanuel Ott
}

\begin{abstract}
Anhand der kompositorischen Probleme, die sich bei der Konzeption mehr als zweistimmiger Kanons ergeben, wird der Frage nachgegangen, wie der konkrete Kompositionsprozess um 1500 ausgesehen haben könnte. Eine Analyse von Jean Moutons Motette Nesciens mater zeigt, dass die Wechselwirkungen zwischen Kanonkonzeption, Klangfolge und melodischer Gestaltung eine simultane Kompositionsweise voraussetzen, in der die Klangfolge wechselnde Verhältnisbestimmungen als Resultat und Voraussetzung der kontrapunktischen Konfiguration erfährt: Der Wechsel von der Sukzessiv- zur Simultankonzeption erscheint in Bezug auf komplexe Kanonbildungen als ein verfahrenstechnisches Erfordernis.
\end{abstract}

Eine bislang offene Frage in Bezug auf die Musik der Renaissance betrifft den realen Kompositionsprozess: Haben Komponisten ihre Stücke Stimme für Stimme - also sukzessiv - oder simultan entwickelt? Gab es überhaupt Möglichkeiten, polyphone Komplexe von vornherein als Ganzes zu konzipieren und zu notieren? Schließlich: War die Klangfolge das `Nebenprodukt der sukzessiven Schichtung einzelner Stimmen oder konnte sie ein selbständiges, die kontrapunktische Konzeption regulierendes Moment bilden? Über diese Themen schweigen sich die Musiktheoretiker des 15. und 16. Jahrhunderts weitgehend aus, wenngleich beispielsweise Markus Jans im Anschluss an Guilielmus Monachus' De preceptis artis musicae demonstriert hat, dass es für Note-gegen-NoteSätze Möglichkeiten gab, mehrstimmige Fakturen quasi algorithmisch aus einer einzigen Stimme herzuleiten. ${ }^{1}$ Auch die auf Edward Lowinsky zurückgehende These eines Paradigmenwechsels von einer sukzessiven zu einer simultanen Kompositionsweise, die er aus einem Passus in Pietro Aarons Libri tres de institutione harmonica ableitet ${ }^{2}$, lässt sich kaum an konkreten Kompositionen nachweisen, da sich dem fertigen Stück schwerlich Hinweise auf den Kompositionsprozess entnehmen lassen.

Folgerichtig untersucht Jessie Ann Owens Komponistenautographen und kommt zu dem Schluss, dass bis ins 16. Jahrhundert hinein sukzessiv komponiert wurde:

1 Vgl. Jans 1986 und 1992.

2 Vgl. Lowinsky 1946. 
[...] the process of composition was essentially additive, lines added to lines, segments to segments. The hypothesis of a dichotomy between successive and simultaneous composition as an explanation for the shift from cantus-firmus or line-based music to imitative music does not fit the evidence of the autograph manuscripts. ${ }^{3}$

Owens' Ergebnis ist umso erstaunlicher, als um 1500 unzählige Kanons verfasst worden sind. Gerade für diese und vergleichbare imitatorische Satzarten wurde lange Zeit angenommen, sie müssten gänzlich oder zumindest partiell simultan (in Partitur oder in Stimmpaaren) angefertigt worden sein: »Imitation, of course, entails working on two parts simultaneously. " $^{4}$ Allerdings finden sich in historischen Traktaten Hinweise, die nahelegen, dass die handwerklichen Voraussetzungen der Kanonbildung auch im Rahmen eines sukzessiven kompositorischen Verfahrens gegeben sind. Beispielsweise basieren die Anleitungen zur Improvisation von zweistimmigen Strettakanons in den Traktaten von Zarlino ${ }^{5}$ und Sancta Maria ${ }^{6}$ darauf, zu einem bestimmten Einsatzintervall zwischen Führungs- und Folgestimme (beispielsweise einer Oberquinte) eine genau definierte Menge an melodischen Intervallen aufzulisten. Wird eine Melodie nur mit diesen Intervallvortschreitungen konstruiert, so ist sie in jedem Falle kanontauglich. Umgekehrt sind alle anderen Intervalle für die Kanonbildung unbrauchbar. Die Vorteile dieses Verfahrens liegen auf der Hand, denn auf diese Weise kann ein Kanon ausgehend von der Einzelstimme, also genuin melodisch erfunden werden.

In Übereinstimmung mit den historischen Traktaten kann so die Entstehung eines großen Teils von Werken, die Kanons inkorporieren, von satztechnischer Warte aus zufriedenstellend als sukzessiver Vorgang erklärt werden: Zweistimmige Kanons, die unzähligen Motetten und Chansons als Gerüst zu Grunde liegen, wären demnach zunächst melodisch konzipiert worden. Freie Stimmen ließen sich diesem Gerüst nachträglich hinzufügen. Bis dahin ist also für den kompositorischen Prozess weder eine Partitur noch ein Konzept von `Akkord oder gar `Harmonikı notwendig.

Allerdings sind aus der Renaissance Kanons überliefert, deren Komplexität die Frage nach dem kompositorischen Vorgehen erneut aufwirft. Gerade in Hinblick auf die Frage, wie Abfolgen von Klängen komponiert und kontrolliert werden konnten, ist eine kleine Gruppe von Quadrupelkanons interessant, die spezifisch französisch zu sein scheint und bisher in der Literatur nicht diskutiert worden ist. Die Gruppe beginnt mit zwei Motetten Jean Moutons (Tabelle 1). ${ }^{7}$

3 Owens 1997, 313.

4 Blackburn 1987, 218.

5 Zarlino 1589, Kapitel III/63.

6 de Sancta Maria 1991, 2. Teil, 208-223.

7 Festas Werk scheint dabei aus der Reihe zu fallen. Edward E. Lowinsky nimmt allerdings unter anderem wegen der Bezüge von Inviolata, integra et casta zu Moutons Nesciens mater an, dass Festa ein Schüler von diesem war (1968, Bd. 1, 49). 


\begin{tabular}{|c|c|c|c|}
\hline Komponist & Titel & $\begin{array}{l}\text { Einsatzabstand } \\
\text { [Mensuren] und } \\
\text {-intervall }\end{array}$ & Erster Nachweis \\
\hline Jean Mouton & Ave Maria gemma virginum & 2 M., Oberoktave & Attaignant 1534/35 \\
\hline Jean Mouton & Nesciens Mater virgo virum & 2 M., Oberquinte & Attaignant 1534/35 \\
\hline Nicolas Gombert & $\begin{array}{l}\text { Qui ne I'aymeroit } \\
\text { (auch als Kontrafaktur } \\
\text { O lesu Christe) }\end{array}$ & 2 M., Unterquarte & $\begin{array}{l}\text { Kriesstein »Selectissimae } \\
\text { necnon familiarissimae } \\
\text { cantiones«, Augsburg } 1540 \\
\left(\text { RISM } 1540^{7} \text { ) }\right.\end{array}$ \\
\hline \multirow[t]{4}{*}{ Costanzo Festa } & Inviolata, integra et casta & & \multirow{4}{*}{$\begin{array}{l}\text { Rom (Città del Vaticano), } \\
\text { Biblioteca Apostolica } \\
\text { Vaticana, Capella Sistina, } \\
\text { Ms. } 20 \text { (ca. 1534-1545) }\end{array}$} \\
\hline & Prima Pars & 4 M., Oberoktave & \\
\hline & Secunda Pars & 3 M., Oberquinte & \\
\hline & Tertia Pars & 1 M., Oberquarte & \\
\hline Philippe Verdelot & Qui la dira la peine & 2 M., Einklang & Livre de Meslanges (1560) \\
\hline
\end{tabular}

Tabelle 1: Liste von Quadrupelkanons

\section{Simultan oder sukzessiv?}

Doppelkanons wurden um 1500 vielfach komponiert; allein von Josquin gibt es fünf. ${ }^{8}$ Quadrupelkanons lassen sich von ihrer Anlage her als Erweiterung von Doppelkanons begreifen. Für Kombinationen mehrerer zweistimmiger Kanons kann nicht mehr eine einzige Führungsstimme den Ausgangspunkt bilden, sondern nur ein mindestens zweistimmiger >Führungssatzı. Es stellt sich folglich die Frage, ob Komponisten bei der Verfertigung solcher Stücke ebenfalls sukzessiv vorgingen, also einen zweistimmigen Kanon nach dem anderen verfassten, oder ob die Führungsstimmen als kompletter mehrstimmiger Führungssatz en bloc entworfen wurden.

Die erste Annahme scheinen Werke zu stützen, in denen einem bereits bestehenden mehrstimmigen Kanon weitere Kanons hinzugefügt wurden. Ein berühmtes Beispiel ist der Tripelkanon Basies moy ${ }^{9}$, in dem Josquins Doppelkanon um einen dritten zweistimmigen Kanon ergänzt wurde. Eine weniger bekannte, aber ungleich spektakulärere Erweiterung findet sich in der >Bearbeitung « von Jean Moutons vierstimmigem Kanon En venant de Lyon durch Pieter Maessins (um 1505-1562). ${ }^{10}$ Maessins lagert in diesem Stück zwei weitere vierstimmige und einen zweistimmigen Kanon sowie zwei freie Stimmen an Moutons Chanson an, so dass ein insgesamt 16-stimmiger Satz entsteht.

In beiden Werken ist allerdings ein wichtiges kompositorisches Regulativ bereits durch die Kanons vorgegeben, nämlich die Klangfolge. So sind die Vorlagen bereits für

8 Die vier Chansons Baisiez moy (NJE 28.4), Dictez moy bergere (NJE 28.10), En I'ombre d'ung buissonnet (NJE 28.13) und Se congié prens (NJE 28.31) sowie die Motette Salve regina (NJE 25.4).

9 des Prés 1922, 51-52.

10 Maessins 1995, 85-94. 
sich genommen vollständige vierstimmige Werke. Sollen einem bestehenden Kanon weitere Kanons hinzugefügt werden, kann leicht bestimmt werden, welche Töne die neu komponierten Stimmen zu einem bestimmten Zeitpunkt erreichen dürfen. Dementsprechend finden sich in den erwähnten Stücken abweichende Klänge zwischen Vorlagen und den erweiterten Werken nur an Stellen, an denen die Vorlagen geringstimmig sind. Insofern besteht die kompositorische Aufgabe bei der Ergänzung eines mehrstimmigen Kanons um weitere Kanons vor allem darin, eine neue melodische Linie durch die vorgegebenen Klänge zu legen.

Für die Gruppe der Quadrupelkanons bleibt dieses Problem jedoch bestehen. Diese Kanons gelingen nicht alleine dadurch, dass die einzelnen Führungs- und Folgestimmenpaare für sich genommen jeweils einen satztechnisch fehlerfreien zweistimmigen Kanon bilden, sondern die zweistimmigen Kanons müssen auch zusammen einen korrekten achtstimmigen Satz ergeben. Diese Aufgabe wird dadurch erschwert, dass die Folgestimmen, die intervallisch und zeitlich gegen die Führungsstimmen verschoben sind, deren weiteren Verlauf limitieren: Sobald die Folgestimmen eingesetzt haben, können in den Führungsstimmen nur solche Töne gewählt werden, die sich mit den Folgestimmen sinnvoll ergänzen. So liegt es nahe anzunehmen, dass die Führungsstimmen in diesen Stücken ein integrales Ganzes bilden, einen `Führungssatzı, nicht eine Summe von vier Führungsstimmen.

Dass die Klangfolgen eines Quadrupelkanons kontrolliert werden müssen, impliziert allerdings nicht, dass die Komponisten beim Anfertigen der Werke rakkordisch gedacht hätten. Wie zahlreiche Traktate belegen, war es aber möglich, von einem Intervall zwischen zwei Stimmen ausgehend eine Art sTonvorrat‘ zu bestimmen, der angibt, welche Töne in den weiteren Stimmen eingesetzt werden können. ${ }^{11}$ Wird darüber hinaus auch der Ton einer dritten Stimme festgelegt, kann eine vierte Stimme wiederum nur ganz bestimmte Töne ergreifen. So finden sich etwa folgende Anweisungen in Pietro Aarons Toscanello von 1529:

Quando il tenore sarà nel unisono col canto, poni il tuo có[n]trobasso in quinta sotto del tenore: \& il tuo alto in terza ó in ottaua, \& (sel ti piace) in decima di sopra al basso: $\&$ quello che sarà a te piu al proposito $[\ldots] .^{12}$

Es gab also keine Notwendigkeit, Klänge als primäre Einheiten aufzufassen, da sie über Intervallrelationen zwischen allen beteiligten Stimmen beschrieben werden konnten.

Anhand von Moutons Marien-Motette Nesciens mater virgo virum lässt sich diese Denkweise gut nachzuvollziehen. Die beiden Moutonschen Quadrupelkanons unterscheiden sich deutlich hinsichtlich ihres satztechnischen Anspruchs. So ist Ave Maria gemma virginum mit einer Länge von 36 Mensuren nur etwa halb so lang wie Nesciens mater und als Kanon in der Oberoktave verfasst. Die Kontrolle der Klangfortschreitun-

11 Vgl. Owens 1997, 24-33.

12 »Wenn der Tenor im Einklang mit dem Cantus ist, setze deinen Contrabassus eine Quinte unter den Tenor und deinen Altus eine Terz, eine Oktave oder (wenn es dir beliebt) eine Dezime über den Bassus, je nach dem, was am angemessensten erscheint.« (Aaron 1529, II, Kap. 21, „Del modo del comporre il controbasso, et controalto doppo il tenore et canto, precetto primo«, eigene Übersetzung) 
gen fällt bei diesem Einsatzintervall leichter als bei der Quinte, die in Nesciens mater das Kanonintervall bildet. In der zuletzt genannten Komposition erhöhen sich die kompositorischen Schwierigkeiten zusätzlich dadurch, dass der Tenor der Motette aus dem gleichnamigen Choral abgeleitet ist (Beispiel 1). Wollte man annehmen, dass Mouton zuerst den satztechnisch einfacheren Kanon verfasst hat, wäre Nesciens mater als das später entstandene Werk anzusehen.
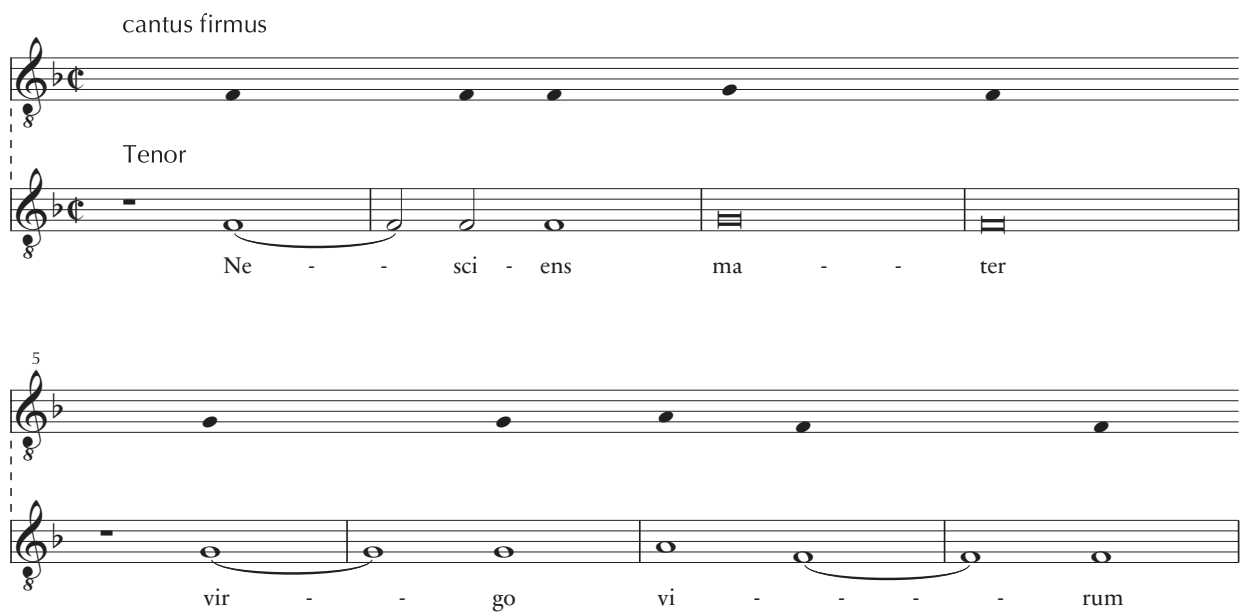

Beispiel 1: Jean Mouton, Nesciens mater virgo virum, Choral und Anfang der Tenorstimme

Die Festlegung der Parameter seines Kanons muss im Hinblick auf die Beschaffenheit des cantus firmus erfolgen. Mouton dürfte also in einem ersten Schritt den cantus firmus als Kanon eingerichtet und in einem zweiten Schritt die übrigen Stimmen angefertigt haben.

\section{Der Cantus-firmus-Kanon}

Vor allem durch die Rhythmisierung der Choralmelodie lässt sich die Intervallfolge zwischen Führungs- und Folgestimme so koordinieren, dass ein satztechnisch fehlerfreier Kanon entsteht. Eine Schwierigkeit besteht allerdings darin, dass sowohl das Einsatzintervall wie auch der zeitliche Einsatzabstand über den gesamten Verlauf des Stückes konstant bleiben. So kann es vorkommen, dass die gewählten Parameter am Anfang eines Stückes zu korrekten Lösungen, an späteren Stellen jedoch zu Problemen führen. In diesem Fall kann sich ein Komponist damit behelfen, den cantus firmus in einzelne Phrasen zu zerlegen, Pausen in die Melodie einzufügen oder von der Vorlage abzuweichen.

Obwohl für das Verhältnis zwischen Führungs- und Folgestimme prinzipiell alle Intervalle in Frage kommen, gibt es eine Präferenz für Quinte, Quarte und Einklang bzw. Oktave. Der Abstand wird dabei sinnvollerweise im Hinblick auf den melodischen Verlauf des cantus firmus gewählt. So eignet sich der Choral Nesciens mater wegen seiner zahlreichen Tonwiederholungen und Sekundschritte am ehesten für Quart- oder Quint- 
kanons, während eine Einrichtung als Einklangs- oder Oktavkanon genau deswegen problematisch ist. (Sekundschritte führen in eng imitierenden Einklangs- oder Oktavkanons regelmäßig zu Sekund- bzw. Septdissonanzen.) Sollte der Choral dennoch als Einklangsoder Oktav-Kanon eingerichtet werden, müsste die Folgestimme später, nach den ersten Sekundschritten der Melodie einsetzen. Dabei käme es allerdings im Zusammenklang vielfach zu Unisoni bzw. Oktaven und in rhythmischer Hinsicht zu unorganischen Mensurteilungen (Beispiel 2).

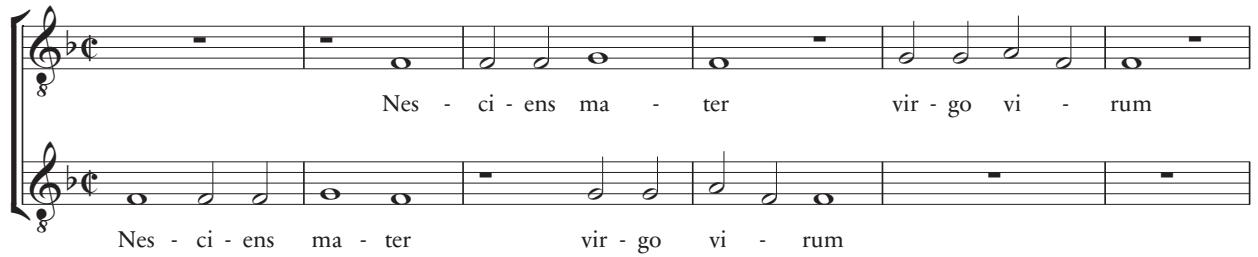

Beispiel 2: Eine mögliche Einrichtung des Choral-Anfangs als Einklangskanon

Aber auch aus einer Einrichtung der Melodie als Oberquart- oder Oberquintkanon resultieren Probleme. So rufen in einem Oberquartkanon die Tonwiederholungen der Melodie die Quarte als Zusammenklang hervor; in einem Oberquintkanon gilt dasselbe für steigende Sekundschritte der Melodie. Diese Quarten bedürfen in einem späteren Arbeitsschritt der `Konsonantmachung` durch fundierende Töne des Basses bzw. Contratenors.

Neben der Bestimmung des Einsatzintervalls des Kanons muss der Komponist festlegen, in welchem zeitlichen Abstand die Folgestimme einsetzen soll. Für einen Oberquintkanon ist dabei einzig ein Strettakanon ausgeschlossen, da der fallende Terzsprung
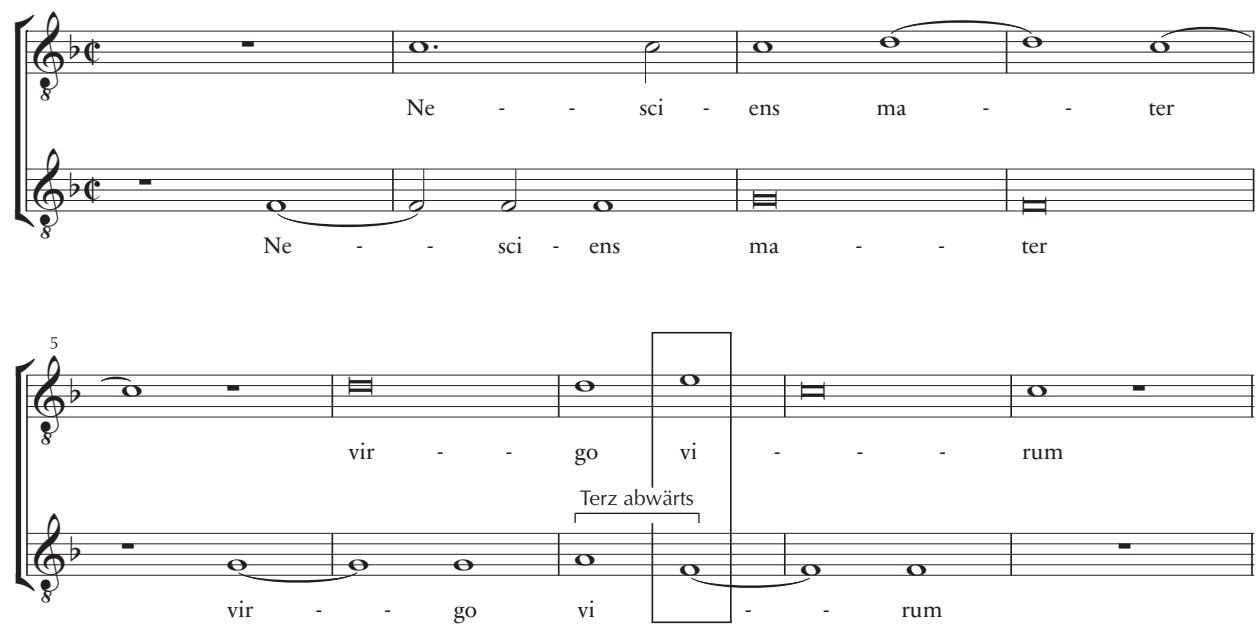

Beispiel 3: Bei der Einrichtung als Strettakanon entstehende Dissonanz

18 | ZGMTH 9/1 (2012) 
am Ende der ersten Phrase in dieser Konstellation zu einer Septimendissonanz führte (Beispiel 3).

Mouton hat also zunächst einen verhältnismäßig großen Spielraum bei der Wahl der grundlegenden Parameter des Kanons. Allerdings haben die einmal festgelegten Parameter erheblichen Einfluss auf die konkrete Ausgestaltung der Tenorstimme im weiteren Verlauf. So ist beispielsweise die Pause am Anfang von Mensur 5 notwendig, um eine Quintparallele zwischen Führungs- und Folgestimme zu vermeiden (Beispiel 4).
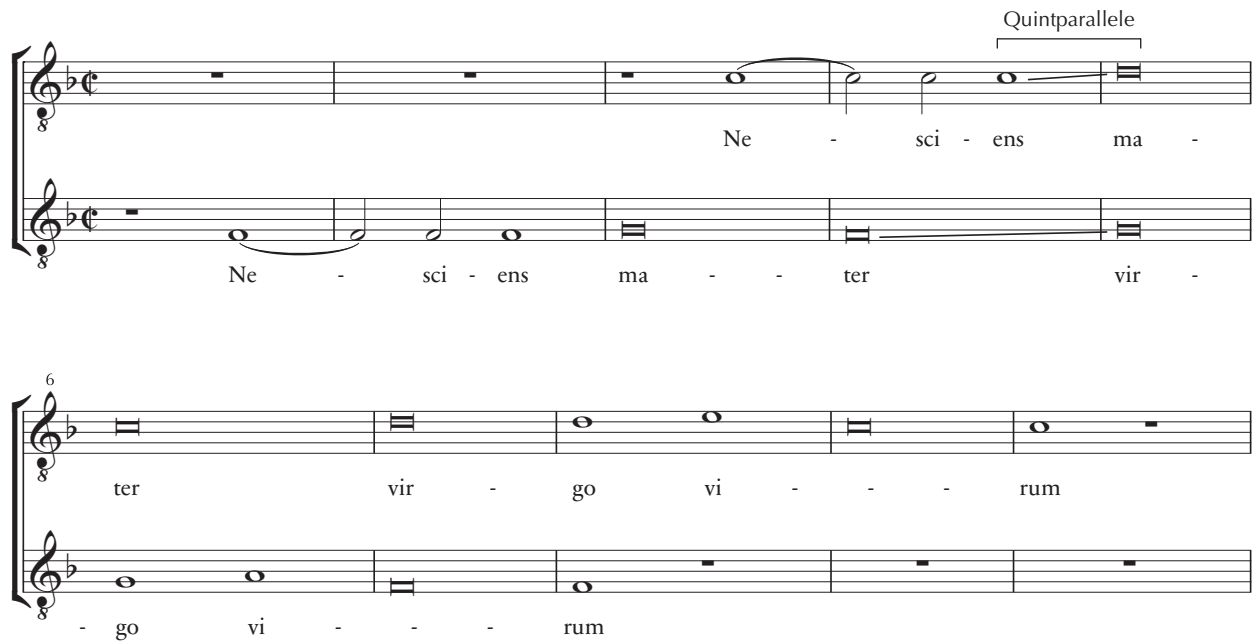

Beispiel 4: Ohne Pause entstehende Quintparallele

Später im Stück resultieren aus der einmal getroffenen Entscheidung für bestimmte Kanon-Parameter noch weitreichendere Schwierigkeiten. So muss Mouton beispielsweise in Mensur 19 einen Ton der Choralvorlage ändern, um eine fehlerhafte Dissonanzenbildung zu vermeiden. Hier ersetzt er den durch den Choral vorgegebenen Ton a durch eine Wiederholung des Tons $b$ (Beispiel 5).

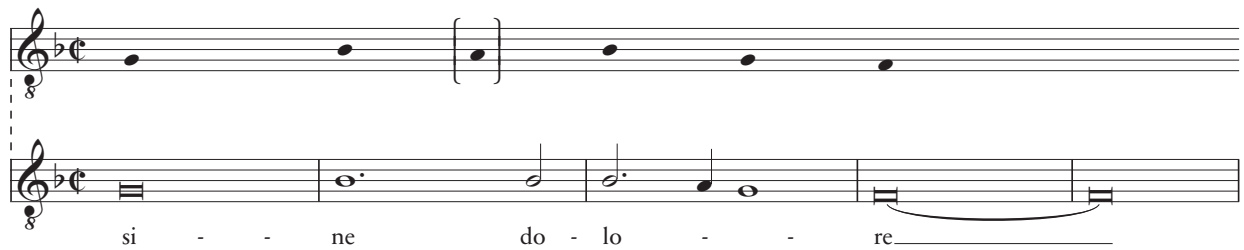

Beispiel 5: Abweichung von der Choralvorlage

Hätte Mouton das a belassen, so wäre bei gleich bleibender Rhythmisierung eine Septime zwischen dem Tenor und seiner Folgestimme entstanden (Beispiel 6). 


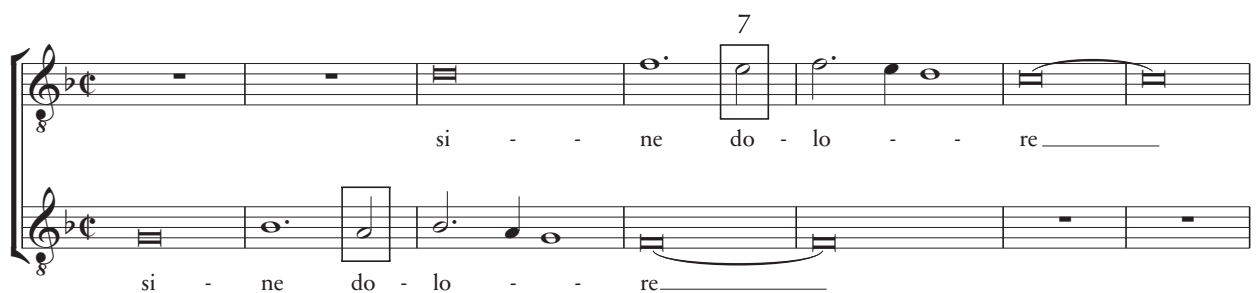

Beispiel 6: Ohne die Abweichung entstehende Dissonanz

\section{Die Klangfortschreitungen}

Durch die Festlegung, den Choral als zweistimmigen Oberquintkanon mit einem zeitlichen Abstand von zwei Mensuren einzurichten, hat Mouton zugleich die Bedingungen für alle anderen Stimmen festgelegt. Demnach werden alle Töne des Führungssatzes zwei Mensuren später in der Oberquinte erscheinen. Angenommen, der Satz begänne mit einem Terz-Quint-Klang auf $f$, so erschiene dieser Klang zwei Mensuren später als ein Terz-Quint-Klang auf c. Sowohl in den übrigen Stimmen als auch im Führungssatz könnten nur die in diesem Klang enthaltenen Töne eingesetzt werden. ${ }^{13}$ Würde dieses 'Reaktionsmuster regelmäßig fortgeführt, so ergäbe sich - dem Einsatzintervall des Kanons entsprechend - eine Kette von Klängen im Oberquintabstand (Beispiel 7).

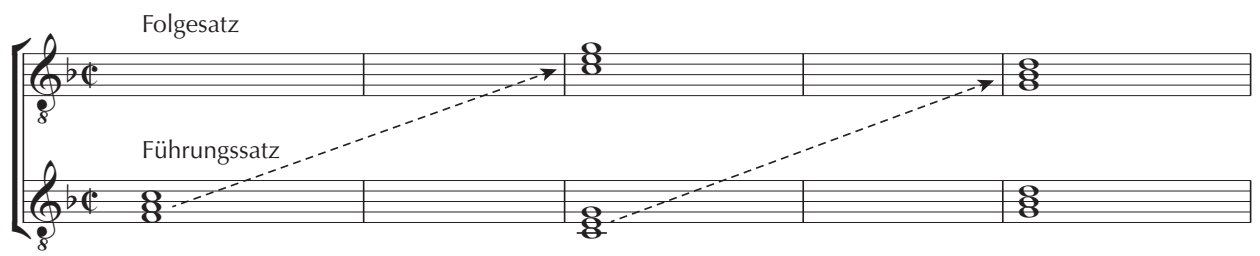

Beispiel 7: Durch den Kanon entstehende Kette von Klängen im Oberquintabstand

Um aus dieser stereotypen Klangfolge ausbrechen zu können, muss der Komponist sich eines Kunstgriffs bedienen. Durch drei unterschiedliche Töne ist ein Zusammenklang vollständig bestimmt. Wird die Anzahl der verschiedenen Töne jedoch auf zwei oder gar einen reduziert, so können die verbleibenden Töne in einen neuen klanglichen Kontext eingebunden werden. Dort, wo im Führungssatz bloß zweistimmige (also ıunvollständige`) Klänge erscheinen, hat der Komponist die Möglichkeit, die Klangfolge in eine andere Richtung zu wenden. Würden beispielsweise zu Beginn nur die Töne $f$ und a eingesetzt, so erklängen nach zwei Mensuren im Folgesatz die Töne $c$ und e, die statt durch ein $g$ auch durch ein a ergänzt werden könnten. Damit ergäbe sich eine andere Quintenkette (Beispiel 8).

13 Im Folgenden wird stets von Terz-Quint-Klängen gesprochen. Damit ist immer ein Tonvorrat im oben erwähnten Sinne gemeint. Natürlich ließen sich alle hier beschriebenen Beziehungen zwischen Klängen auch in Form von Tabellen nach dem Vorbild etwa Pietro Aarons darstellen. 


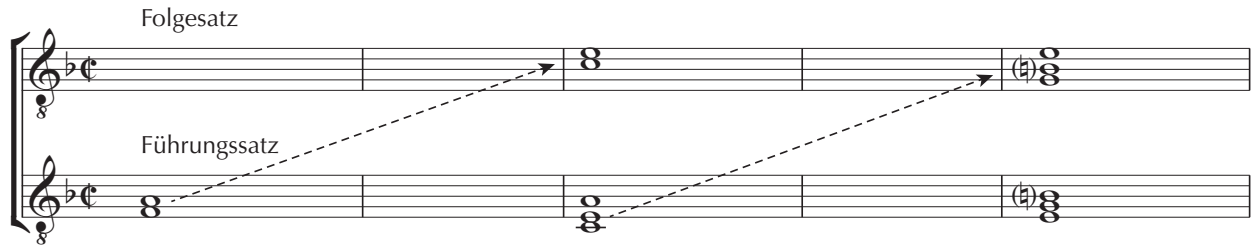

Beispiel 8: Umlenkung der Klangfortschreitung durch alternative Ergänzung eines zweistimmigen Klanges

Die reichsten Umdeutungsmöglichkeiten entstehen selbstverständlich, wenn im Führungssatz nur ein einzelner Ton eingesetzt wird. Erscheinen etwa im Verlauf des Stückes im Folgesatz die Töne $f$-a-c, so steht es dem Komponisten frei, welche Töne er verdoppelt. Abhängig von der Auswahl der Töne kann von einem Klang aus in fünf verschiedene Klänge fortschritten werden (Beispiel 9).

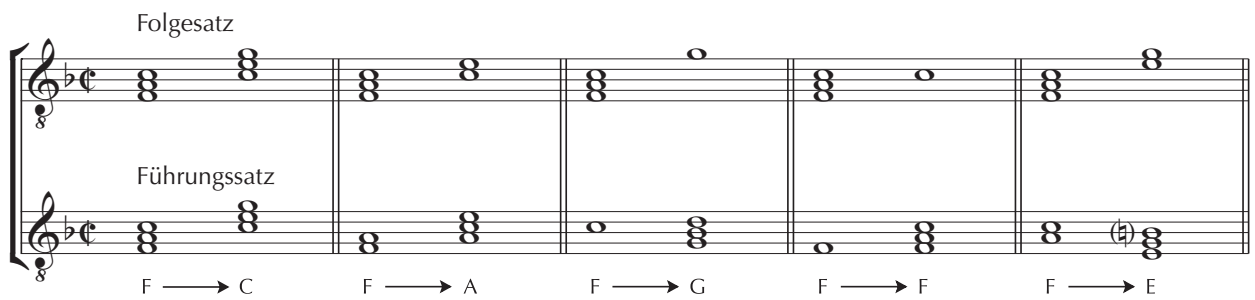

Beispiel 9: Möglichkeiten der Klangfortschreitung

Somit kann der Komponist bereits im Vorfeld genau bestimmen, welche Töne der vierstimmige Führungssatz erreichen darf, damit zwei Mensuren später ein gewünschter Klang entstehen kann. Allerdings sind bestimmte Klangverbindungen durch das Kanonintervall ausgeschlossen. So gibt es beispielsweise keine Möglichkeit, auf einen $f$-Klang zwei Takte später einen $d$-Klang folgen zu lassen. Solche gezielten Manipulationen im Dienste bestimmter Klangfortschreitungen lassen sich in der gesamten Motette Moutons nachweisen. Dies sei exemplarisch an einer kurzen Passage dargestellt (Beispiel 10).

Unterschieden werden muss zwischen den im gesamten achtstimmigen Satz entstehenden Klängen und den Tönen, die im Führungssatz eingesetzt werden. Die Klänge des gesamten Satzes, die auf den ersten drei Semibreven des Ausschnitts entstehen, stehen zu ihren jeweils zwei Mensuren später entstehenden Pendants im Quintabstand: Den zwei Terz-Quint-Klängen auf $g$ folgen zwei Klänge auf $d$, während der c-Klang auf der ersten Semibrevis in Mensur 36 zwei Mensuren später einen Terz-Quint-Klang auf $g$ zur Folge hat. Eine erste Abweichung von diesem Schema findet sich in dem darauf folgenden $d$-Klang, der zwei Mensuren später nicht in einen erwarteten a-, sondern einen $c$-Klang mündet. Mouton kann hier entsprechend nur die Töne $f$ und a im Führungssatz einsetzen. Bemerkenswerter noch ist die Gestaltung der Klänge zwischen den Mensuren 37 und 39, da hier jeweils derselbe Klang entsteht, eine Verbindung, bei der im Füh- 
Folgesatz (Fuga in dapenthe)

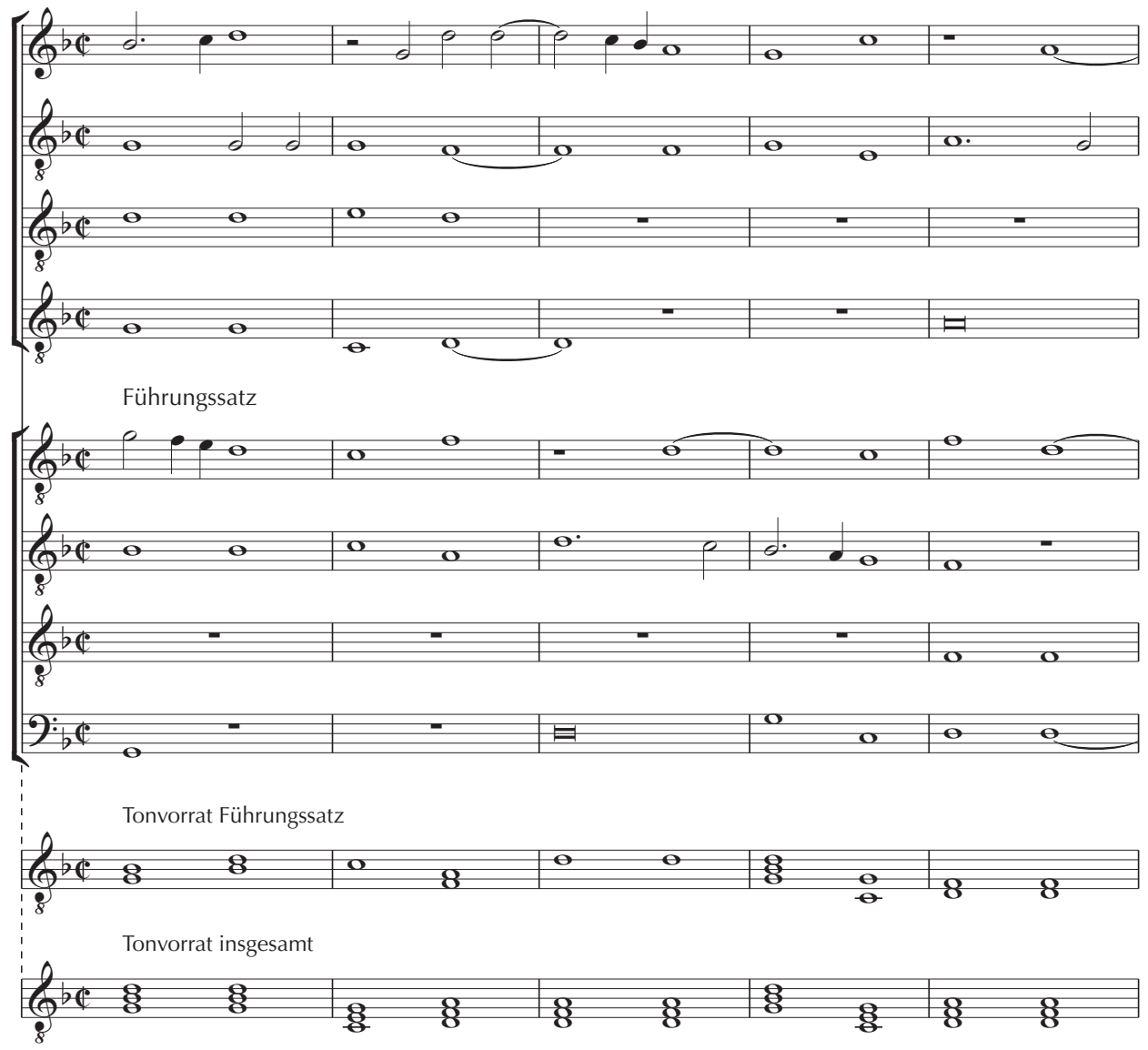

Beispiel 10: Jean Mouton, Nesciens mater, M. 35-39

rungssatz nur ein einziger Ton erscheinen darf. Statt in einer solchen Situation diesen Ton nur in einer einzigen Stimme einzusetzen und andere Stimmen erst später hinzutreten zu lassen, lässt Mouton ihn in drei Stimmen gleichzeitig erscheinen.

Deutlich wird an diesem Ausschnitt aber auch, wie sensibel Mouton ästhetisch mit den Erfordernissen des kompositorischen Verfahrens umgeht. So erscheinen im Führungssatz selbst Klänge, die zwei Mensuren später in der Oberquinte erscheinen - also dem `Reaktionsmuster entsprechen -, kaum je vollständig. Die Unvollständigkeit eines Klangs ermöglicht daher noch keine Vorhersage, ob von ihm aus eine neue Richtung eingeschlagen wird, oder ob sein Pendant wiederum in der Oberquinte erscheinen wird. 
Nesciens mater stellt fraglos einen Höhepunkt der Kanontechnik am Anfang des 16. Jahrhunderts dar. Dies zeigt sich sowohl darin, dass spätere Werke nach diesem Vorbild gearbeitet sind, als auch in der Erwähnung der Motette in den Traktaten von Glarean, Zarlino und Cerone. Die Autoren weisen allerdings vor allem auf deren achtstimmige Struktur hin und erwähnen nur en passant, dass es sich bei ihr um einen Kanon handelt. Dabei ist es gerade diese kompositorische Machart, die das Stück in vielen Aspekten definiert, und die Aufgabe, einen Quadrupelkanon über einen cantus firmus zu verfassen, hinterlässt Spuren in jedem Aspekt des Satzes.

Nicht zuletzt zeugt Nesciens mater damit von einer sich verändernden Kompositionspraxis: Während für vierstimmige Sätze um 1500 bestenfalls unterstellt werden kann, dass Komponisten eine Vorstellung von der sharmonischen Entwicklung، eines Stückes hatten, lässt sich für Moutons Motette zeigen, dass die Möglichkeit, in Klangfortschreitungen zu denken, essentiell für das Gelingen der kompositorischen Aufgabe war.

\section{Noten}

Des Prés, Josquin (1922), Werken van Josquin des Prés, hg. von Albert Smijers, »Wereldlijke Werken«, Serie 22, Heft 1, 51-52.

Maessins, Pieter (1995), Sämtliche Werke (= Denkmäler der Tonkunst in Österreich 149), hg. von Othmar Wessely und Martin Eybl, Graz, 85-94.

\section{Literatur}

Aaron, Pietro (1529), Toscanello in Musica, Venedig: Bernardino et Matheo de Vitali, Reprint Bologna: Forni 1999 (= BMP II/10).

Blackburn, Bonnie (1987), „On Compositional Process in the Fifteenth Century«, Journal of the American Musicological Society 40/2, 210-284.

de Sancta Maria, Fray Thomas (1565), Libro Llamado El Arte de tañer Fantasía, übers. von Almonte C. Howell, Jr. und Warren E. Hultberg als The art of playing the fantasia, Pittsburgh: Latin American Literary Review Press 1991.

Jans, Markus (1986), »Alle gegen Eine. Satzmodelle in Note-gegen-Note-Sätzen des 16. und 17. Jahrhunderts", in: Basler Jahrbuch für historische Musikpraxis 10, 101-120.

(1992), »Modale sHarmonikı. Beobachtungen und Fragen zur Logik der Klangverbindungen im 16. und 17. Jahrhundert", in: Basler Jahrbuch für historische Musikpraxis 16, 167-188.

Lowinsky, Edward E. (1946), »The Concept of Physical and Musical Space in the Renaissance «, in: Papers of the American Musicological Society. Annual Meeting 1941, 57-84.

(1968), The Medici Codex of 1518. A Choirbook of Motets Dedicated to Lorenzo de' Medici, Duke of Urbino, 3 Bde., Chicago: University Press.

Owens, Jessie Ann (1997), Composers at Work. The Craft of Musical Composition 14501600, New York: Oxford University Press.

Zarlino, Gioseffo (1558), Istitutioni harmoniche, Venedig: Appresso Francesco Senese. 